\title{
Denoising of Atomic-Scale Images Based on Automatic Grain Segmentation, Unsupervised Primitive Unit Cell Extraction and Periodic Block-Matching
}

\author{
Benjamin Berkels ${ }^{1}$ and Niklas Mevenkamp ${ }^{1}$ \\ 1. AICES Graduate School, RWTH Aachen University, Schinkelstr. 2, 52062 Aachen, Germany.
}

Nowadays electron microscopes are commonly used to acquire images at atomic resolution [1]. However, for many relevant materials, the resolution is not limited by the microscope's physical properties, but by the beam sensitivity of the material. The beam sensitivity limits the electron dose that can be applied before the observed material is damaged. In turn, the electron dose limits the achievable signal-to-noise ratio (SNR) and thus the effective resolution. Hence, for beam sensitive materials, it is crucial to extract as much information from the available data as possible, which can be done by leveraging prior knowledge.

Typically, the SNR is improved by either a kind of local averaging or by averaging multiple images of the same specimen. Unfortunately, the former reduces the effective spatial resolution while the latter increases the necessary electron dose. The key observation to improve the SNR without reducing the effective spatial resolution or increasing the electron dose is that images at atomic resolution contain many self-similar image regions due to the underlying atomic grid. The self-similarities allow for non-local averaging, i.e. the averaging of similar image patches where the similarity does not rely on the spatial proximity of the patches (like in local averaging methods) but on the similarity of the intensities in the patches.

One of the most popular modern non-local denoising algorithms for digital photographs is the Blockmatching and 3D filtering algorithm (BM3D) [2]. Due to the very different structure of digital photographs and electron micrographs, BM3D does not make full use of the self-similarities in atomic scale images. To take advantage of the special features of atomic-resolution electron micrographs of crystals, we proposed a specially tailored denoising strategy based on BM3D [6]. It uses a block-matching strategy that exploits the periodic structure of the observed crystals and is designed to cope with strong Poisson noise that is typical for low dose electron micrographs. On simulated single shot Scanning Transmission Electron Microscopy (STEM) images of inorganic materials, this method achieves a considerably higher peak SNR and a better precision than the original $\mathrm{BM} 3 \mathrm{D}$, which makes no periodicity assumptions on the input.

An important ingredient for the denoising algorithm [6] is sufficiently accurate knowledge of the primitive unit cell, i.e. a unit cell that can't be further simplified, of the depicted crystal. In [4], we proposed a novel method for the unsupervised estimation of the primitive unit cell from crystal images. Candidate lattice vector angles are obtained following [8] from significant peaks of the projective standard deviations of the input image. For each angle estimate, a corresponding fundamental period estimate is determined by clustering local minima of a periodicity energy. Here, the number of clusters is determined in an unsupervised manner using likelihoods of multi-variance cluster models. Finally, the primitive unit cell is defined by the two vectors of minimal fundamental period (with corresponding angle estimates), which are then refined jointly using non-linear optimization. The resulting algorithm can robustly estimate complex primitive unit cells from both synthetic and experimental images, even on images with low SNR.

A limitation of the denoising algorithm [6] is its periodicity assumption on the input micrograph, i.e. the original formulation cannot handle micrographs that show materials with discontinues in the periodicity pattern like they would appear, for instance, in case of multiple grains. Inside each periodic region though, 

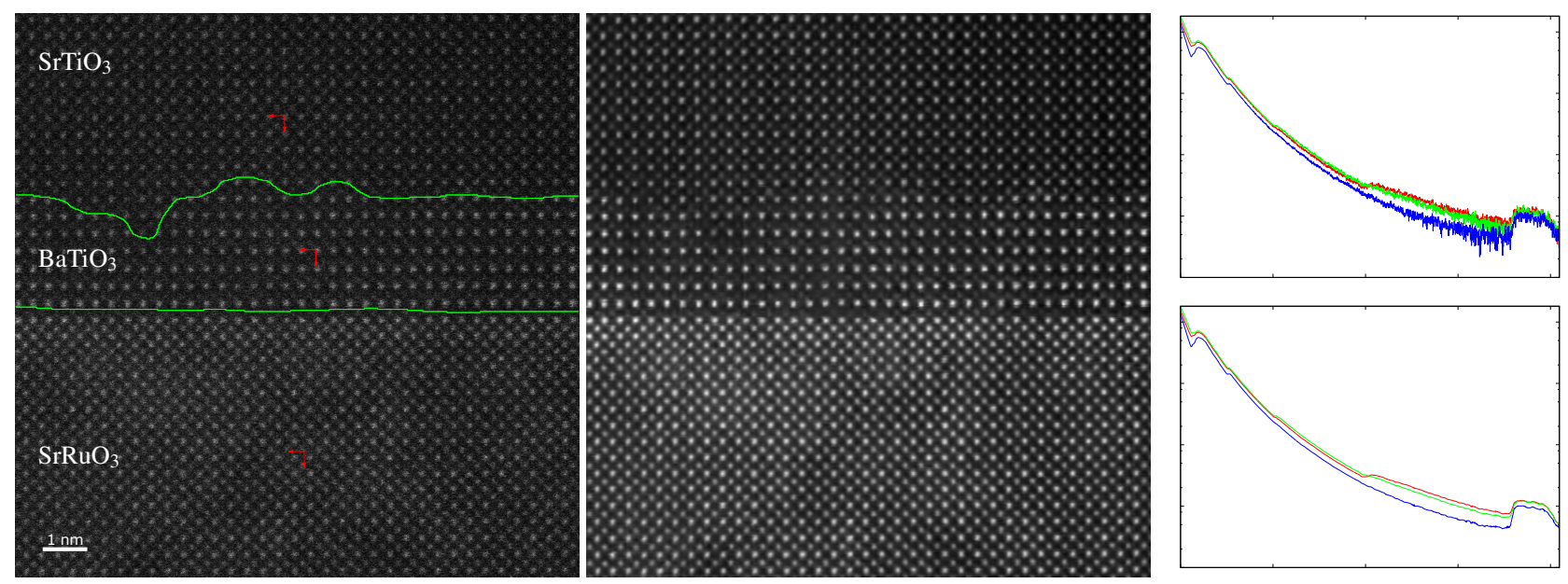

Figure 1. STEM image [7] (left) with automatically detected grains (boundaries in green) and unit cells (red), and denoising result from the non-local adaptive periodic BM3D approach (middle). Right: Three spectra of an EELS data set [3] and the denoised spectra obtained by applying BM3D on the whole data set.

[6] can be applied. To this end, we developed a segmentation algorithm for crystal images that is able to handle extremely noisy input data [5]. It uses a novel method for multi-phase segmentation of images based on high-dimensional local feature vectors. Localized Fourier transforms are used to construct feature vectors that encode periodicity information. The segmentation is built around the multi-phase Mumford-Shah model and a projection of the high dimensional features onto a low-dimensional space using principle component analysis. The former provides a regularization on the boundary length of the segments, while the latter is essential since the initialization of the high-dimensional mean features directly is infeasible.

Combining [5], [4] and [6] allows the denoising of crystal images with multiple grains: First the grains are detected using [5]. Then, [4] is used to extract the primitive unit cell of each grain. Finally, each grain is denoised exploiting its periodicity using [6]. In contrast to the Poisson noise model used in [6], we use a mixed Poisson-Gaussian noise model that applies to a wider range of micrographs and is directly applicable to the raw data from the microscope. Fig. 1 illustrates the procedure on a STEM image from [7].

BM3D is not limited to classical images or micrographs, but also very suitable for hyperspectral imaging techniques, like electron energy loss spectroscopy (EELS). Instead of comparing local image patches to find similar regions for the non-local averaging, the spectra can be compared directly and without periodicity assumptions. A denoising result on an EELS data set from [3] is shown in the right part of Fig. 1.

[1] PE Batson, N Dellby, and OL Krivanek, Nature 418 (2002), pp. 617-620.

[2] K Dabov et al., IEEE Transactions on Image Processing 16.8 (2007), pp. 2080-2095.

[3] M Duchamp et al., Applied Physics Letters 102.13 (2013).

[4] N Mevenkamp and B Berkels, GCPR Proceedings (2015), pp. 105-116.

[5] N Mevenkamp and B Berkels, WACV Proceedings (2016).

[6] N Mevenkamp et al., Advanced Structural and Chemical Imaging 1 (2015).

[7] D Park et al., Microscopy and Microanalysis 20 (2014), pp. 740 -747.

[8] X Sang and JM LeBeau, Ultramicroscopy 138 (2014), pp. 28 -35.

[9] The authors acknowledge funding from the Excellence Initiative of the German Federal and State Governments and thank D. Park and M. Duchamp for providing STEM and EELS data, respectively. 